\title{
Optimization in collaborative information systems for an enhanced interoperability network
}

\author{
Aicha Koulou ${ }^{1, *}$, Maria Zemzami ${ }^{1,2}$, Norelislam El Hami ${ }^{1}$, Abir Elmir ${ }^{3}$, and Nabil Hmina ${ }^{1}$ \\ ${ }^{1}$ LGS Lab - ENSA, Ibn Tofail University, Kenitra, Morocco \\ ${ }^{2}$ LITIS Lab - INSA, Normandy University Rouen, Rouen, France \\ 3 ARDT Lab - ENSIAS, Mohammed V University, Rabat, Morocco
}

Received: 7 March 2019 / Accepted: 21 October 2019

\begin{abstract}
Today's constraints on collaborative enterprises are often directly reflected in the interoperability of their information systems. The goal is to improve and effectively evolve the global interoperability of collaboration by distributing the required effort in an optimal way on different information systems to achieve the target goal. This method of enhancement will be realized by applying an IMA (Interoperability Measurement Approach) to generate a ratio representing the degree of interoperability of an information system within the collaboration, as well as applying the heuristic optimization method PPSO (Paralleled Particle Swarm Optimization) to reach the optimal distribution of effort.
\end{abstract}

Keywords: Optimization / interoperability / IMA / PSO / healthcare network

\section{Introduction}

In terms of exchange and collaboration between companies, internally and externally, interoperability (and more specifically the interoperability of information systems) is now considered as a major challenge for fostering collaborative activity and to develop the performance of the collaborative network.

Generally speaking, interoperability is conceived as the ability of two systems to understand one another and to mutually use their functionality. The word "inter-operate" implies that one system performs an operation for the other one. From the computer technology point of view, it is the faculty for two heterogeneous computer systems to function jointly and to give access to their resources in a reciprocal way [1].

According to the literature, there are various definitions of interoperability which were presented by different researchers in different sectors and domains. We quote the two most significant to our context. The first one is "the ability of two or more systems or elements to exchange information and to use the information that has been exchanged". The second definition is "The capability promoted but not guaranteed - achieved through joint

\footnotetext{
* e-mail: aicha.k2007@gmail.com
}

conformance with a given set of standards, that enables heterogeneous equipment, generally built by various vendors, to work together in a network environment' [2].

To improve interoperability inside collaborative network, it is necessary to analyze and diagnose the current situation in order to be able to identify any problems that might occur, as well as opportunities for improvement $[3,4]$.

The premise of the present work is to optimize the distribution of efforts on all interconnected information systems in order to enhance and evolve the global interoperability, and thereby increase the level of the collaborative network. This paper is organized as follows: Section 2 displays an overview of the main steps of IMA (Interoperability Measurement Approach) [5], Section 3 proposes a modeling of interoperable information systems, Section 4 describes the heuristic optimization method adopted in the present article, and finally, Section 5 illustrates how to reach optimum effort repartition in multi-project environment across a healthcare collaborative network.

\section{Interoperability measurement approach}

IMA is a method of interoperability assessment. This method is based on the three fundamental aspects of interoperability, compatibility, maturity and performance. The principle of this method is the calculation of the degree 
Table 1. Interoperability compatibility (proposed by [12]).

\begin{tabular}{|c|c|c|c|c|c|c|}
\hline & \multicolumn{2}{|c|}{ Conceptual } & \multicolumn{2}{|c|}{ Organizational } & \multicolumn{2}{|c|}{ Technology } \\
\hline & Syntactic & Semantic & $\begin{array}{l}\text { Authorities } \\
\text { responsibilities }\end{array}$ & Organization & Platform & Communication \\
\hline Business & cq11 & cq12 & cq13 & cq14 & cq15 & cq16 \\
\hline Process & cq21 & cq22 & cq23 & cq24 & cq25 & cq26 \\
\hline Service & cq31 & cq32 & cq33 & cq34 & cq35 & cq36 \\
\hline Data & cq41 & cq42 & cq43 & cq44 & cq45 & cq46 \\
\hline
\end{tabular}

Table 2. Quantification of the maturity of the interoperability.

\begin{tabular}{ll}
\hline Maturity level (IMML) & Potentiality quantification \\
\hline 1 & 0.2 \\
2 & 0.4 \\
3 & 0.6 \\
4 & 0.8 \\
5 & 1 \\
\hline
\end{tabular}

of each of the three preceding aspects, then the generation of the global degree of interoperability according to the four following steps:

\subsection{Quantifying the compatibility degree}

In this step, we define the incompatibilities of various nature between the interoperable enterprises, and this by identifying, for each concern of the enterprise (data, service, process and business), all the barriers that influence the proper functioning of the interoperability [6-9]. Barriers to interoperability can be classified into three categories:

- conceptual barriers: encompass syntactic and semantic incompatibilities related to information exchanged between interoperable enterprises/systems,

- organizational barriers: represent barriers related to human and organizational behavior (the definition of responsibility and authority),

- technological barriers: represent the incompatibilities of the information technologies used by the different partners. These incompatibilities concern the presentation, processing and communication of data.

The incompatibility is identified using a questionnaire and based on a modified version of the matrix proposed by $[10,11]$ (see Tab. 1). If any incompatibilities are detected, the value 0 is given; conversely, the value 1 will be assigned if no incompatibility is identified.

And the degree of compatibility is calculated by the following formula:

$$
C=\sum_{i} \sum_{j} \frac{c q_{i j}}{24}
$$

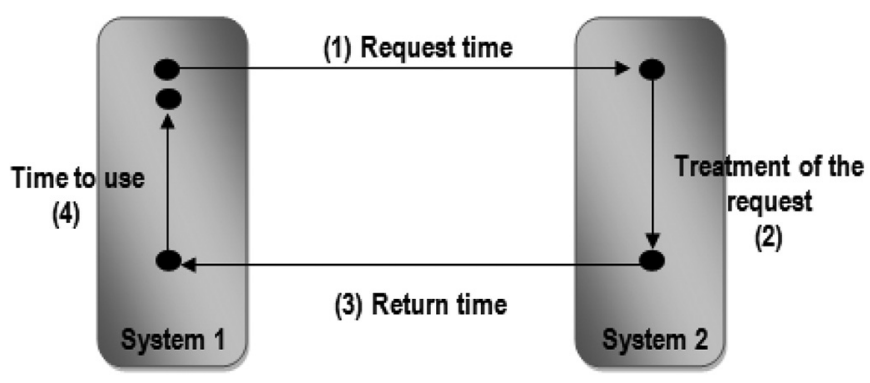

Fig. 1. Decomposition of the time of interoperability (proposed by [5]).

\subsection{Evaluating maturity}

This step consists of evaluating the degree of potentiality of an enterprise/system to interoperate with one or more partners whose identity is not known. This assessment requires the adoption of a model among the existing interoperability maturity models, such as LISI, OIMM, EIMM, LCIM... etc. [13,14]. The principle of these models is to classify the system /enterprise into one of the five defined maturity levels. Thus, the degree of maturity is obtained by adopting the mapping proposed by [10] (see Tab. 2).

\subsection{Calculating performance degree}

Quantification of the degree of interoperability performance is achieved by the geometric mean of the following three degrees: degree of time of interoperability (TI), degree of cost of interoperability (CI) and degree of quality of interoperability (QI) (see the formula (2)):

$$
P O=\sqrt[3]{(T I \times Q I \times C I)}
$$

\subsubsection{Degree of time of interoperability}

The time of interoperability is decomposed into several periods of time (see Fig. 1). The value of each period of time is represented by a ratio between the real time and that provided by the Service-Level Agreement (SLA). And finally, the degree of time of interoperability is defined by the geometric mean of all previous values of periods of time. 


\subsubsection{Degree of quality of interoperability}

The quality of interoperability contemplates three kinds of quality, the quality of exchange $\left(q_{e x}\right)$, the quality of conformity $\left(q_{\text {conf }}\right)$ and the quality of use $\left(q_{\text {use }}\right)$. The degree of quality of interoperability is defined through the geometric mean of the previous kinds (see formula (3)).

$$
Q I=\sqrt{\left(q_{e x} \times q_{\text {conf }} \times q_{u s e}\right)} .
$$

\subsubsection{Degree of cost of interoperability}

The cost of interoperability is characterized by the cost of exchange $\left(c_{e x}\right)$ and the cost expected to make the information exchanged usably $\left(c_{u s e}\right)$.

The value of each two previous costs is achieved by comparing the cost of reference (SLA) and the real cost. Therefore, the degree of cost of interoperability is defined as:

$$
C I=\sqrt{\left(c_{e x} \times c_{u s e}\right)} .
$$

\subsection{Aggregating the interoperability degree}

The overall degree of interoperability can be obtained by the arithmetic mean (see formula (5)):

$$
I M A=\frac{(P I+D C+P O)}{3} .
$$

If the IT department has elements to ponder each of these three indicators with different weights $\left(n_{1}, n_{2}, n_{3}\right)$, we choose the weighted arithmetic mean (see formula (6))

$$
I M A=\frac{\left(n_{1} * P I+n_{2} * D C+n_{3} * P O\right)}{\left(n_{1}+n_{2}+n_{3}\right)} .
$$

\section{Systems interconnection modeling}

Improvement and upgrading of a collaborative network is achieved through the execution of changes and the application of efforts at the level of interconnected information systems. In this paper, we view the collaborative network as a multi-project environment that focuses on achieving a defined goal. In such an environment, the allocation of resources and the distribution of effort, with the aim of upgrading collaboration, refer to a challenge of optimizing the overall effort and distributing it in a multiproject of implementation of interoperability $[15,16]$.

In this section, we propose a coupling of the IMA approach with linear modeling in order to characterize the evolution of the overall degree of interoperability of a set of interconnected information systems. Consider $n$ systems $\left(S_{1}, S_{2}, \ldots \ldots, S_{n}\right)$, we associate with each system $S_{n}$ a ratio $a_{i}=$ IMA $\left(S_{i}\right)$ representing its degree of interoperability. The vector $\left(a_{1}, \ldots, a_{n}\right)$ advances as per the exertion made to adjust the internetworked system from current As-is state to the objective To-Be state as far as the undertaking design vision. By denoting:

$V=\left(a_{i}\right)$ represents the current vector.

$V^{\prime}=\left(a_{i}^{\prime}\right)$ represents the planned interoperability vector.

We have for each system $S_{i}$ :

$$
a_{i}^{\prime}=\sum_{j} E_{i j} * a_{j}
$$

$E_{i j}$ represents the effort to apply on the $a_{i}$ system in order to improve the $a_{j}$ system.

$E=\left(E_{i j}\right)$ the matrix effort to apply in order to obtain the planned interoperability.

For this situation, we will probably locate the optimal effort to achieve the focused on interoperability vector. In this way, the target function to minimize is:

$$
a_{i}^{\prime}-\sum_{i} \sum_{j} E_{i j} * a_{j} \leq 0
$$

The requirements are for every $j$ :

$$
\sum_{i} E_{i j} \leq 100
$$

To optimize the target function (8) regarding the requirements in (9) it is conceivable to utilize deterministic techniques such as the gradient function. But in the case of large scale systems, these solutions stay ineffective as far as performance.

\section{Parallel particle swarm optimization}

Particle Swarm Optimization (PSO) was first described in 1995 by James Kennedy and Russell C. Eberhart. It is a stochastic evolutionary algorithm based on the population based on socio-psychological principles. It finds a solution to an optimization problem in a search space predicting social behavior in the presence of goals $[17,18]$.

In a PSO algorithm, every particle " $\mathrm{i}$ " is treated as a point in a space with dimension $\mathrm{D}$, a position $\mathrm{X} \mathrm{i}$, a velocity $\mathrm{Vi}$ and an individual best position $\mathrm{X}$ besti. The individual best position related with a particle $\mathrm{i}$ is the best position that the particle has visited. The best position of all particles in the swarm is represented by the vector $\mathrm{X}$ gbest $[19,20]$. The flowchart of the PSO algorithm is given in Figure 2.

$\mathrm{X}$ i : represents the initial particle;

$\mathrm{V}$ i : represents the initial velocity of the particle;

$\mathrm{X}$ besti: defines the best position of particle;

$\mathrm{X}$ gbest: defines the global best position of the swarm.

The techniques have considerably evolved and the original version of the algorithm is barely recognized in the current ones. In this paper, we opt for the Parallel Particle Swarm Optimization. The latter is an improvement of the basic PSO algorithm by using a new evolutionary neighborhood concept associated with parallel computing.

According to the author of [21], the use of Dynamic Neighborhood makes it possible to better explore and 


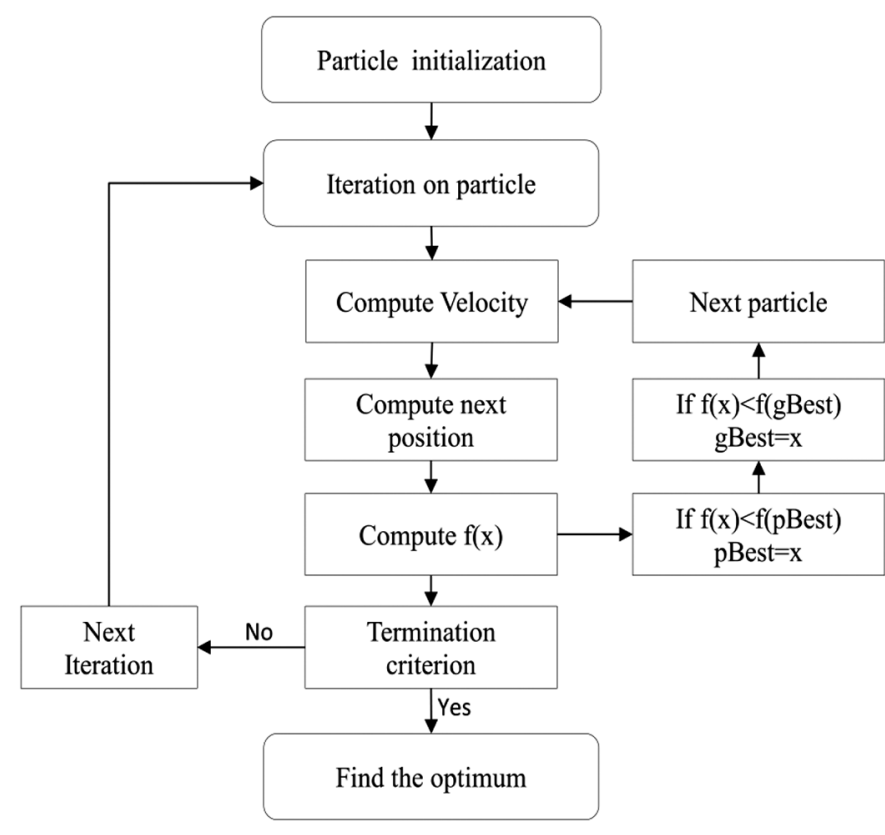

Fig. 2. Flowchart of the classic PSO algorithm.

exploit the search space by updating neighborhoods at each iteration of the algorithm. For example: a particle i which has place with a group N5 at the iteration N115 is declared best of its group; then at the iteration N116, this particle i has a place with another group N9 and offers its information with its new group: this segment improves the movement of its new group and speed up the movement of the other particles towards the optimum.

Furthermore, the use of parallelization concepts minimizes computational time, principally for complex optimization problems. Parallelization is based on the use of threads (a mechanism to manage parallel execution workflows). A set of threads are created during every iteration, each thread is responsible for processing the PSO for its group (neighborhood). The search for the optimum is done in parallel for all groups. Below is the flowchart of the model used (Fig. 3):

\section{Case study and results}

\subsection{Case study description}

Nowadays, healthcare sector is experiencing a remarkable increase in the use of information systems. It uses SI which are structured especially around the medical record to support the storage, access, dissemination and sharing of patient information. But these SI are characterized by autonomy, distribution and heterogeneity. For that reason, the integration of IT with healthcare system requires interoperability that may lead to open connectivity at all levels (i.e. inpatient and outpatient care) [22].

Consequently, interoperability in healthcare sector has become an essential preoccupation that fostered many researchers to investigate more to achieve and develop this interoperability. The majority of research is focused on the semantic heterogeneity and development of standards in order to ensure the working together of various systems for the welfare of patients. However, this research stays defective as it targets only the semantic and standards development issues, i.e., it covers just a small scale. They should explore all levels to find about the weaknesses or barriers that handicap the efficiency of interoperability.

In our illustrative case study, we investigated the degree of interoperability and its consequences on the performance of e-health process, especially in "surgical procedure". This process involves the interconnection of four entities (health service, hospital, radiology, and laboratory) and includes the following nine sub-activities (see Fig. 4):

- Surgery decision-making: after the diagnosis and safety of a surgical necessity, the patient information was recorded.

- Updating personal file: this activity is carried out at the level of the health service to ensure the overall management of patients' files.

- Analysis results: before surgery, the patient needs to undergo prior analyses. Some of these analyses need to be performed in a laboratory. So, the hospital system accesses the laboratory data to view the results.

- Surgical operation: this activity is done at the hospital and provides a report of the surgery.

- Updating personal file: next to surgery, it is planned to interact with the health service to update the personal patient's file.

- Radiology report: after the recovery period, if the patient's case needs radiography, the hospital system consults the radiography report by acceding in radiology data.

- Final surgery report: the last step of this process is to produce a final surgery report and to update the personal patient's file by interacting system of health service.

The main goal of this case study is to illustrate in detail the stages of our proposed method.

\subsection{Evaluation of interoperability with IMA}

The first stage of application of our method is to define the current situation of interconnection between the previous entities (health service, hospital, radiology, and laboratory). For that, we applied the IMA approach to assess, for each entity, each level on its own to detect where the problem exactly lies and to what extent it constitutes a barrier. Table 3 shows the degree of interoperability calculated with IMA for each participating entity to ehealth process "surgical procedure".

For example, the degree of interoperability of health service is calculated as follows (see formula (10)):

$I M A_{\text {HealtServ. }}=\frac{64+58+\sqrt[3]{49 \times 56 \times 75}}{3}=60.34 \approx 60 \%$. 


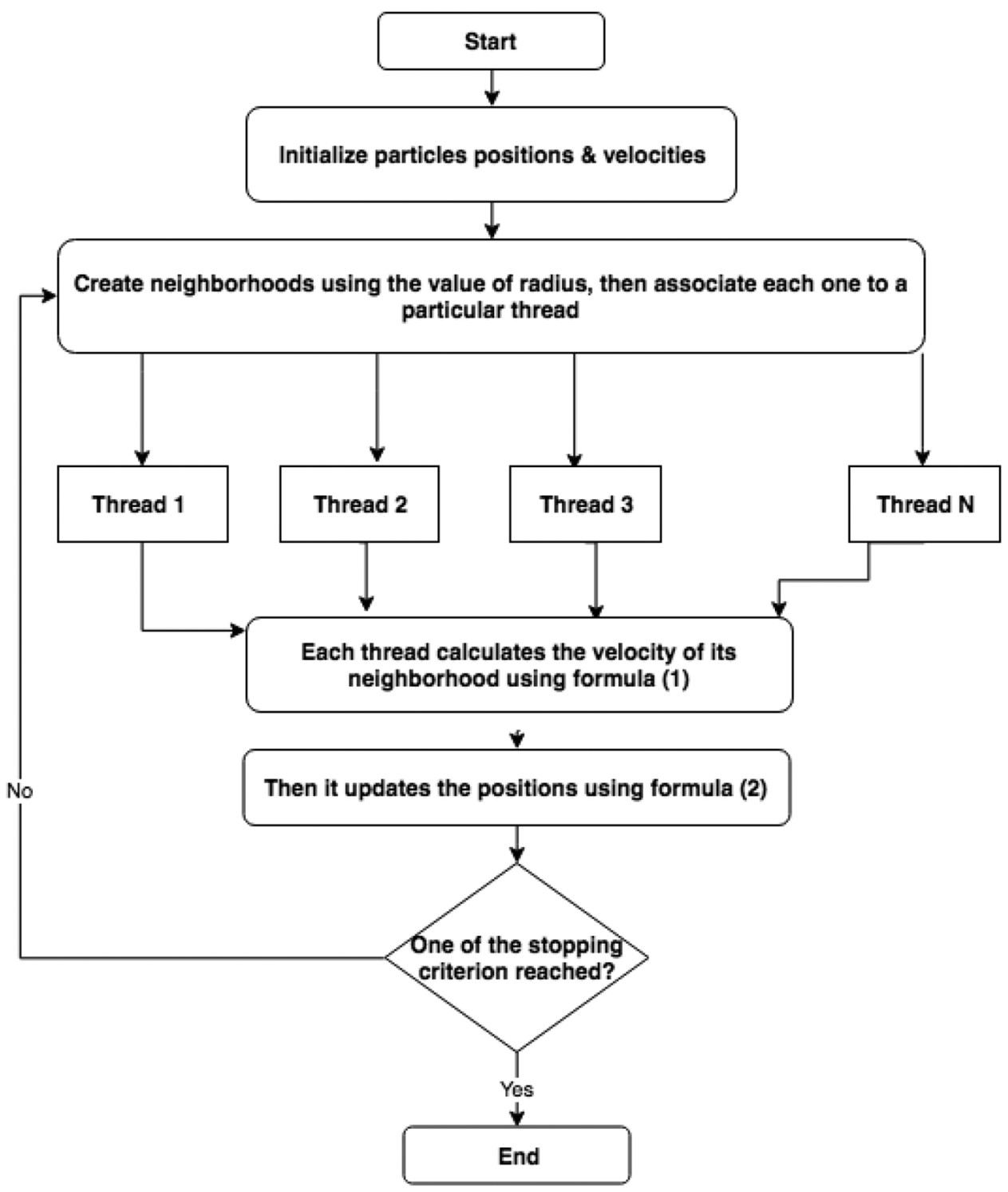

Fig. 3. Flowchart of Parallel PSO algorithm.

Table 3. Degree of interoperability in "surgical procedure" process.

\begin{tabular}{lllllll}
\hline & Potentiality score & Compatibility score & \multicolumn{3}{c}{ Performance } & \multirow{2}{*}{ IMA degree } \\
\cline { 4 - 5 } & & & $\mathrm{TI}$ & $\mathrm{QI}$ & $\mathrm{CI}$ & \\
\hline Health service & $64 \%$ & $58 \%$ & $49 \%$ & $56 \%$ & $75 \%$ & $60 \%$ \\
Hospital & $32 \%$ & $30 \%$ & $37 \%$ & $40 \%$ & $34 \%$ & $33 \%$ \\
Radiology & $45 \%$ & $57 \%$ & $45 \%$ & $39 \%$ & $50 \%$ & $49 \%$ \\
Laboratory & $40 \%$ & $15 \%$ & $28 \%$ & $50 \%$ & $30 \%$ & $30 \%$ \\
\hline
\end{tabular}

\subsection{Modeling and optimization}

After calculating the current degree of interoperability of each participating entity using IMA, the objective of this stage is to evolve the global efficiency of studied process. The decision taken is to improve interoperability degrees according to Table 4 .
In this case, we numbered the previous entities as follows: $\mathrm{S} 1=$ Health service; $\mathrm{S} 2=$ Hospital; $\mathrm{S} 3=$ Radiology center; $\mathrm{S} 4=$ Laboratory. Figure 5 shows the interaction of the four systems within e-health network.

Then, we modeled the evolution of interoperability degree such as shown in Figure 6. 


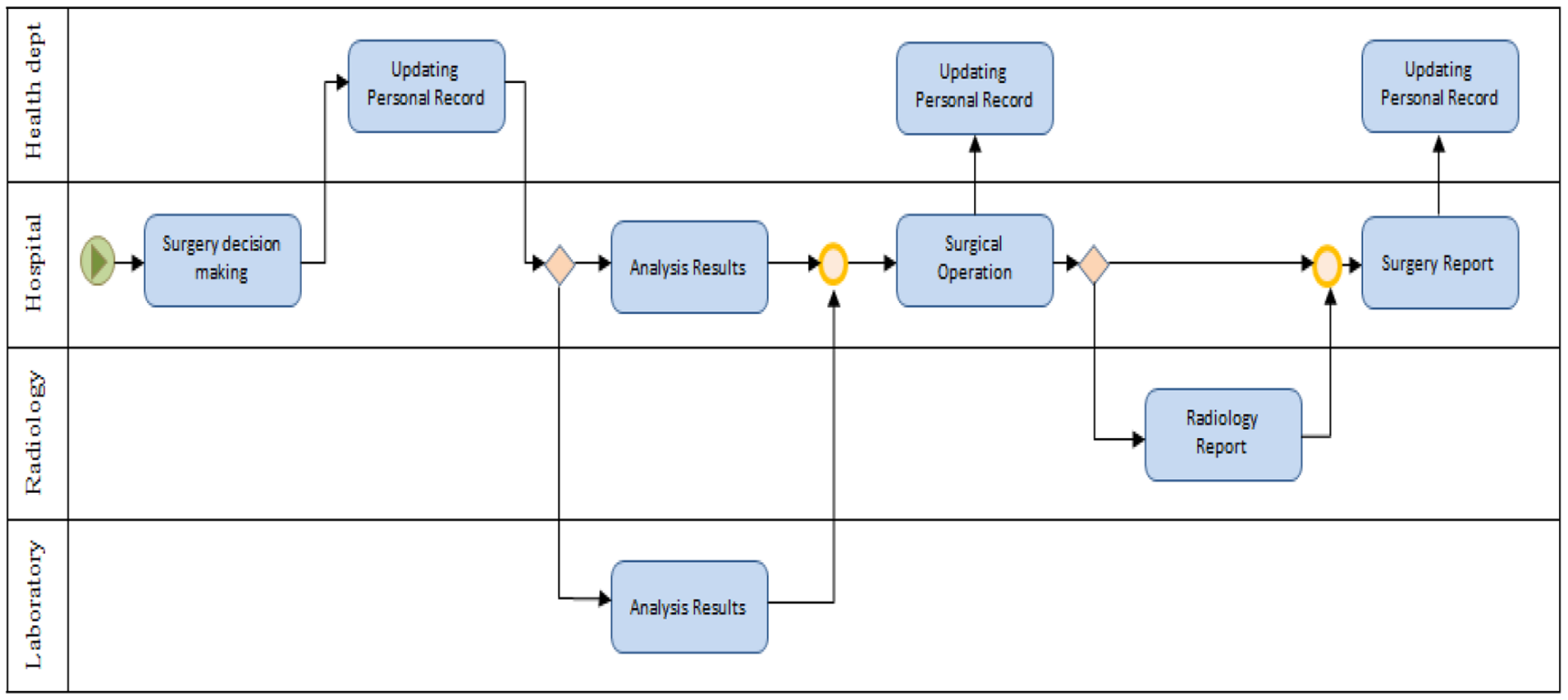

Fig. 4. Collaborative surgical operation process.

Table 4. Plannified improvement of e-health process.

\begin{tabular}{lll}
\hline & $\begin{array}{l}\text { Current IMA } \\
\text { degree }\end{array}$ & $\begin{array}{l}\text { Target IMA } \\
\text { degree }\end{array}$ \\
\hline Health service & $60 \%$ & $63 \%$ \\
Hospital & $33 \%$ & $50 \%$ \\
Radiology & $49 \%$ & $48 \%$ \\
Laboratory & $30 \%$ & $54 \%$ \\
\hline
\end{tabular}

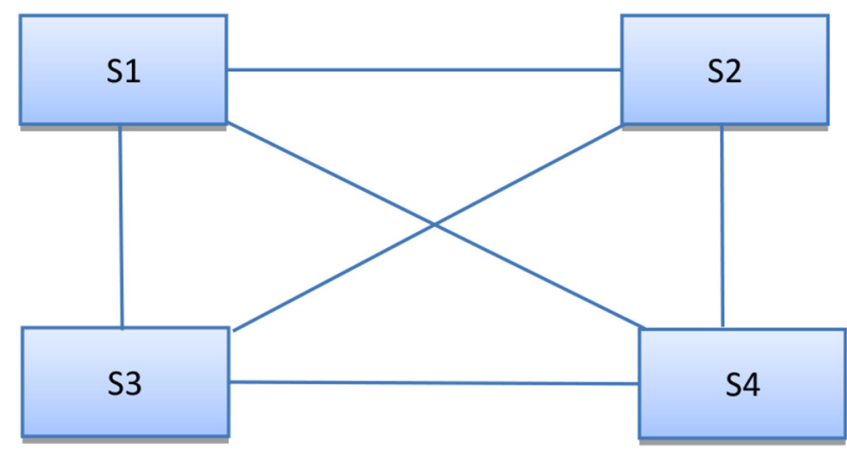

Fig. 5. Interaction model in e-health network.

Eventually, we applied the PPSO algorithm. The use of the PPSO has provided the optimal effort matrix that minimizes the objective function. As mentioned in [23]: "PSO has yielded better results based on combined objective function criteria as compared to other non-traditional optimization methods. It was also observed that PSO converged to the global optimal solution at a faster rate and took less computational time. Though, all the nontraditional optimization methods used in this work are stochastic, PSO is simple in its structure. PSO algorithm is

$\left(\begin{array}{llll}E_{11} & E_{12} & E_{13} & E_{14} \\ E_{21} & E_{22} & E_{23} & E_{24} \\ E_{31} & E_{32} & E_{33} & E_{34} \\ E_{41} & E_{42} & E_{43} & E_{44}\end{array}\right] \cdot\left[\begin{array}{l}60 \\ 33 \\ 49 \\ 30\end{array}\right]=\left(\begin{array}{l}63 \\ 50 \\ 58 \\ 54\end{array}\right]$

Fig. 6. Interaction model in e-health network.

$\left(\begin{array}{cccc}85 & 5 & 20 & 2 \\ 50 & 15 & 20 & 18 \\ 70 & 8 & 25 & 3 \\ 65 & 12 & 17 & 1\end{array}\right)$

Fig. 7. Optimal efforts distribution (via PPSO).

rather simple to code as compared to AGA Adaptive Genetic Algorithm and SA Simulated Annealing"

The result of the estimation of the optimized effort matrix is represented in Figure 7:

\subsection{Results analysis}

The analysis of the matrix obtained via the PPSO algorithm allows us to identify the different efforts to be attributed to the various interfaces of the participated information systems in our studied e-health process "surgical procedure" and also gives us a clear idea of the 


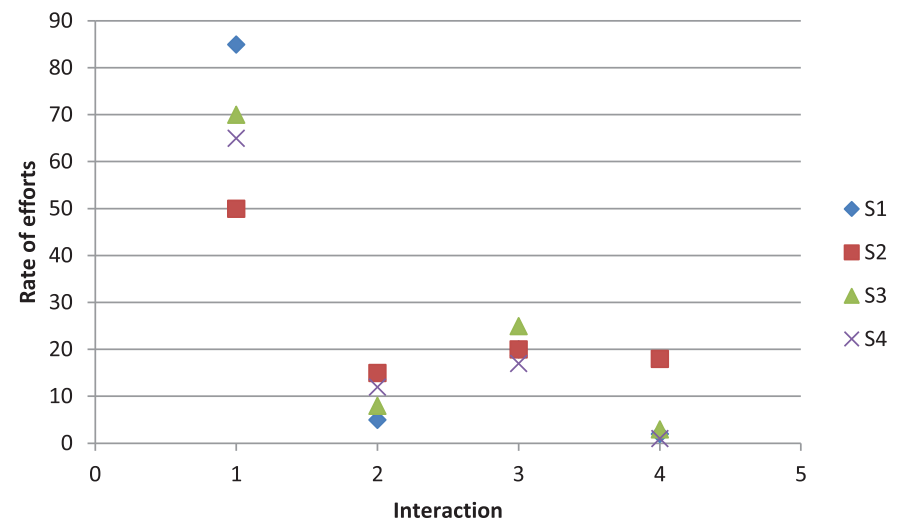

Fig. 8. A graphical representation of the optimal efforts distribution.

strategy to opt in order to achieve the target goal and upgrade the global interoperability of the interconnection within the e-health network.

The graphical representation, as shown by Figure 8, gives us a better visualization of the efforts distribution. Thus, we can note that for the interaction " 1 ", the rates of the necessary efforts are very important for the interactions with $\mathrm{S} 1\left(E_{21}=50 ; E_{31}=70 ; E_{41}=65\right)$ and especially the interaction of $\mathrm{S} 1$ with itself $\left(E_{11}=85\right)$. Therefore, we can say that the most optimal strategy is to focus the efforts mainly on the system of Health service and all the interactions with it.

\section{Analysis}

In this paper, we have presented an efficient approach for interoperability network enhancement. The first stage of application of this approach is to define the current situation of interconnection by assessing the current interoperability degree of each participating entity using IMA. The objective of the second stage is to progress the global efficiency of studied collaboration through modeling the evolution of interoperability degree and applying the PPSO algorithm which provides the optimal effort matrix to be distributed to the various interfaces of the participated information systems (see Fig. 9).

The proposed optimal effort matrix is theoretical and should be compared to the integration architects' proposals for estimating the required effort for the interface of the information systems concerned. Indeed, if the proposed estimate is close to the theoretical result, this proposal can be accepted. Otherwise, the project manager invites the architects to rethink their estimates.

The result may not be realistic in practice, even if it respects the constraints. The result can still be used by integration architects to address the solution. In some cases, the limited optimal outcome may not be practical due to unforeseeable events or lack of human or budgetary resources. However, this optimized solution can be used by integration architects in their planning as a target.

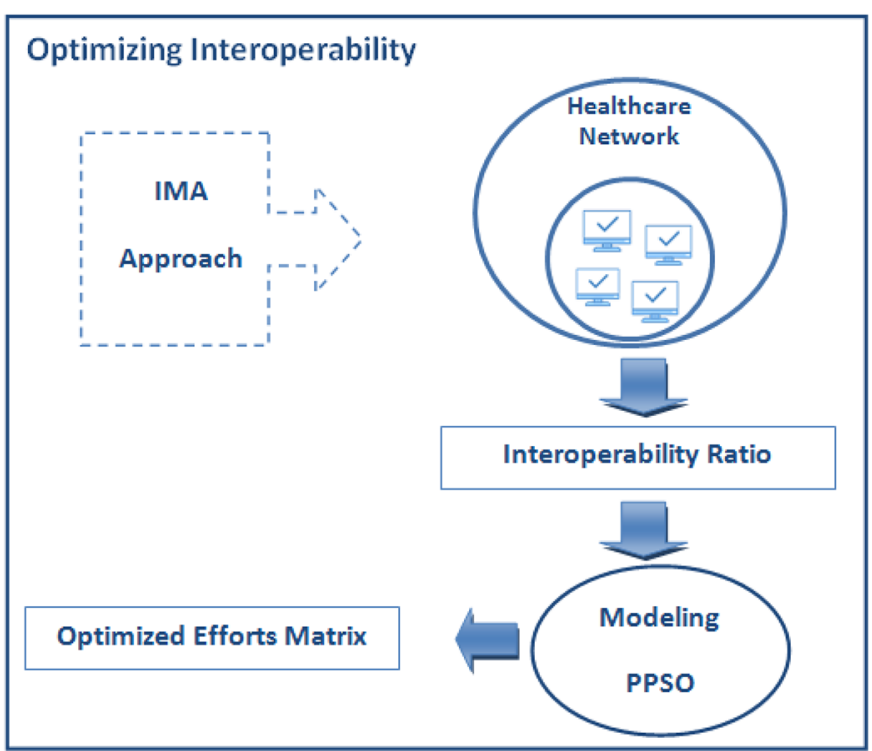

Fig. 9. Interoperability optimization steps.

\section{Conclusion}

The improvement and evolution of the overall interoperability of collaboration is achieved through the application of the necessary efforts. This paper has proposed a model to effectively allocate the necessary efforts in two steps: the generation of IMA ratio representing the degree of interoperability for each information system and the proposal of distribution optimal efforts to establish and improve the interoperability of the collaborative network.

The proposed model is available to be used later by any optimization method to improve the objective function to the defined problem. The optimization operations used in this paper use PPSO parallel particle swarm optimization.

A case study, applied to healthcare collaboration namely "surgical procedure" process, has illustrated the assessment of interoperability using IMA among the network and it has described how interoperability degrees are obtained in each entity. Then, after the modeling, it optimizes an efforts matrix using a PPSO algorithm to obtain the objective.

The results are given by an optimized matrix which allows a good estimate of the effort required for interconnecting the information systems involved.

\section{References}

1. D. Chen, G. Doumeingts, F. Vernadat, Architectures for enterprise integration and interoperability: Past, present and future, Comput. Ind. 59, 647-659 (2008)

2. IEEE 1990. Standard Computer Dictionary: A Compilation of IEEE Standard Computer Glossaries

3. G. Goldkuhl, The challenges of Interoperability in Egovernment: Towards a conceptual refinement, pre-ICIS 2008 SIG eGovernment Workshop, 2008 
4. W. Guédria, Y. Naudet, D. Chen, Maturity model for enterprise interoperability, Enterp. Inf. Syst. 9, 1-28 (2015)

5. A. Koulou, N. El Hami, N. Hmina, A. Elmir, B. Bounabat, A novel approach IMA of interoperability measurement, in 2016 4th IEEE International Colloquium on Information Science and Technology (CiSt), 2016, pp. 665-669

6. D. Chen, B. Vallespir, N. Daclin, An Approach for enterprise interoperability measurement, InMoDISE-EUS, 2008, pp. $1-12$

7. R.A. DeFusco, D.W. McLeavey, J.E. Pinto, M.J. Anson, D.E. Runkle, Quantitative Investment Analysis (John Wiley \& Sons, New York, 2015)

8. T.C. Ford, J.M. Colombi, S.R. Graham, D.R. Jacques, Survey on Interoperability Measurement, Air Force Institute of Technology, OH, 2007

9. N. Daclin, D. Chen, B. Vallespir, Enterprise interoperability measurement-Basic concepts, EMOI-INTEROP, 2006, 6

10. B. Elmir, B. Bounabat, A novel approach for periodic assessment of business process interoperability, Int. J. Comput. Sci. Issues 8, 298 (2011)

11. B. Elmir, B. Bounabat, E-service interoperability measurement within business collaboration networks, in Proceedings of MICS, 2010

12. M. Kasunic, Measuring systems interoperability: Challenges and opportunities, Carnegie-Mellon University, Pittsburgh, PA, Software Engineering Institute, 2001

13. C. Campos, R. Chalmeta, R. Grangel, R. Poler, Maturity model for interoperability potential measurement, Inf. Syst. Manag. 30, 218-234 (2013)
14. G. Doumeingts, D. Chen, Interoperability Development for Enterprise Applications and Software, Cunningham et al. (Eds.), Springer London, London, 2003, pp. 207-214

15. A. Elmir, N. El Hami, B. Elmir, B. Bounabat, R. Ellaia, M. Itmi, Multiobjective optimization of information system quality enhancement, J. Theor. Appl. Inf. Technol. 70, 52-61 (2014)

16. N. El Hami, A. Elmir, B. Elmir, R. Ellaia, M. Itmi, B. Bounabat, Simulation and optimization of interoperability planning, J. Theor. Appl. Inf. Technol. 52 (2013)

17. J. Kennedy, R.C. Eberhart, Particle swarm optimization (McGraw-Hill, New York, 1995)

18. T.R. Ramanan, M. Iqbal, K. Umarali, A particle swarm optimization approach for permutation flow shop scheduling problem, Int. J. Simul. Multidisc. Des. Optim. 5, A20 (2014)

19. N.A. Alrajeh, B. Elmir, B. Bounabat, N. El Hami, Interoperability optimization in healthcare collaboration networks, Biomed. Eng. 57, 403-411 (2012)

20. N. El Hami, R. Ellaia, M. Itmi, Hybrid evolutionary optimization algorithm MPSO-SA, Int. J. Simul. Multidisc. Des. Optim. 4, 27-32 (2010)

21. M. Zemzami, N. Elhami, M. Itmi, N. Hmina, A new parallel approach for the exploitation of the search space based on PSO algorithm, in 2016 4th IEEE International Colloquium on Information Science and Technology (CiSt), 2016, pp. $104-110$

22. U. Batra, S. Sachdeva, S. Mukherjee, Implementing healthcare interoperability utilizing SOA and data interchange agent, Health Policy Technol. 4, 241-255 (2015)

23. K. Babu, Comparison of PSO, AGA, SA and memetic algorithms for surface grinding optimization, Appl. Mech. Mater. 852, 241-247 (2016)

Cite this article as: Aicha Koulou, Maria Zemzami, Norelislam El Hami, Abir Elmir, Nabil Hmina, Optimization in collaborative information systems for an enhanced interoperability network, Int. J. Simul. Multidisci. Des. Optim. 11, 2 (2020) 